\title{
Daily Step Counts in Patients With Chronic Kidney Disease: A Systematic Review and Meta-Analysis of Observational Studies
}

\author{
Fan Zhang ${ }^{1 \dagger}$, Yibo Ren ${ }^{1 \dagger}$, Hui Wang ${ }^{2}$, Yan Bai ${ }^{3}$ and Liuyan Huang ${ }^{1 *}$ \\ ${ }^{1}$ Department of Nephrology, Longhua Hospital Shanghai University of Traditional Chinese Medicine, Shanghai, China, \\ 2 Department of Anorectal, Longhua Hospital Shanghai University of Traditional Chinese Medicine, Shanghai, China, \\ ${ }^{3}$ Department of Cardiology, Longhua Hospital Shanghai University of Traditional Chinese Medicine, Shanghai, China
}

OPEN ACCESS

Edited by:

Yoshiyuki Morishita,

Jichi Medical University Saitama

Medical Center, Japan

Reviewed by:

Wisit Kaewput,

Phramongkutklao Hospital, Thailand

Naoki Nakagawa

Asahikawa Medical University, Japan

Kiyonori Ito,

Jichi Medical University Saitama

Medical Center, Japan

${ }^{*}$ Correspondence:

Liuyan Huang

hly1980@126.com

tThese authors have contributed equally to this work

Specialty section:

This article was submitted to Nephrology,

a section of the journal

Frontiers in Medicine

Received: 23 December 2021

Accepted: 26 January 2022

Published: 17 February 2022

Citation:

Zhang F, Ren Y, Wang H, Bai Y and Huang $L$ (2022) Daily Step Counts in Patients With Chronic Kidney Disease:

A Systematic Review and Meta-Analysis of Observational Studies. Front. Med. 9:842423.

doi: 10.3389/fmed.2022.842423
Background: Physical inactivity is an essential factor in the prognosis of patients with chronic kidney disease (CKD). Daily step count is a straightforward measure to assess physical activity levels. Understanding the step counts among different CKD stages is essential to change sedentary behavior.

Objectives: This systematic review and meta-analysis aimed to investigate the daily step counts in patients with CKD at a different stage.

Design: A systematic review and meta-analysis.

Data Sources: The literature search was performed in PubMed, Embase, and Web of Science from inception to November 3rd, 2021.

Review Methods: Observational studies (cross-sectional, case-control, or cohort studies) reported specific values of step counts in CKD patients by the wearable device were included. A random-effects model was used to pool the data. Subgroup analysis explored differences in outcomes by stage of CKD. Heterogeneity between studies was assessed using the $\chi^{2}$ test of Cochrane's $Q$ statistic. A contour-enhanced funnel plot was conducted to investigate publication bias. Univariate and multivariate meta-regression was conducted to examine possible sources of heterogeneity.

Results: Twenty-eight articles were identified and used for quantitative analysis. The result showed that the daily step count in patients with CKD was 4642.47 (95\% Cl: 4274.18-5010.76), and significantly lower than the healthy population. Subgroup analysis revealed that the step counts decreased before dialysis, dropped to a freezing point at the hemodialysis phase, and increased after kidney transplantation. Meta-regression analysis showed that daily step counts were relatively higher in the Americas or younger than 60 or kidney transplant recipients.

Conclusion: The status of daily step counts in patients with CKD decreases with CKD severity and increases after kidney transplantation. Although studies have begun to focus on strategies to improve step counts in patients with CKD, future studies should focus 
more on step counts in pre-dialysis patients and changing their physically inactive lifestyle early to alleviate deteriorating renal function.

\section{Systematic Review Registration: https://www.crd.york.ac.uk/prospero/display_ record.php?RecordID=291551, identifier: CRD42021291551.}

Keywords: chronic kidney disease, daily step counts, physical activity, systematic review, meta-analysis

\section{INTRODUCTION}

Chronic kidney disease (CKD) has emerged as a major worldwide public health problem, with adverse physical, psychological, and economic outcomes (1). Being physically active is a healthy lifestyle considered a core part of the general and CKD populations (2). A recent Meta-analysis of a cohort study of patients with CKD showed that high physical activity was associated with a $14 \%$ reduction in all-cause mortality compared to low physical activity (3). Correspondingly, the UK Renal Association Clinical Practice Guideline recommends that patients with CKD engage in at least 30 min of moderateintensity physical activity five times per week (4).

Walking is the most common physical activity for chronic diseases, including CKD (5). With the advent of pedometers, it has become feasible in the real world to accurately quantify the number of steps an individual walks each day. The number of daily steps reflects the amount of physical activity an individual is doing (6). Since the use of pedometers in medical research in the 1980s (7), numerous research studies have been conducted on the number of daily steps taken by individuals with CKD.

However, the mean or the median number of steps reported by different populations varies widely. Given the diversity of the results of these studies, this study evaluates the daily step counts in patients with CKD at a different stage by using a systematic review and meta-analysis to determine the differences between subgroups and provide a reference for promoting physical activity in patients with CKD.

\section{METHODS}

This systematic review has been reported according to the Preferred Reporting Items for Systematic Reviews and MetaAnalyses (PRISMA) criteria statement (8). The study protocol was registered with the International Prospective Register of Systematic Reviews (PROSPERO), and the registration number was CRD42021291551.

\section{Search Strategy}

We systematically searched PubMed, Embase, and Web of Science from inception to November 3rd, 2021. Free-text words and medical subject heading $(\mathrm{MeSH})$ terms were used. The whole search strategy is presented in Supplementary Material 1. Moreover, we manually retrieved a relevant reference of systematic reviews to search for additional potential studies.

\section{Eligibility Criteria}

Published observation studies with the following criteria were considered eligible for inclusion in this meta-analysis: (1) participants: CKD patients. There is no limit to age and disease stage (pre-dialysis, peritoneal dialysis, hemodialysis, and kidney transplant recipients were eligible); (2) outcomes: the wearable device to measure the daily steps count; (3) studies: only observational study (cross-sectional, case-control, or cohort studies) designs were included; (4) studies reported specific values [mean (standard deviation, SD), median (quartile or range)] of patient steps were eligible. One literature was excluded if it met at least one of the following criteria: (1) patients affected by acute kidney failure; (2) clinical intervention trials, conference abstract, case reports, protocol, letters, commentaries, reviews, and editorials; (3) written in non-English.

\section{Literature Selection}

Two authors (Yan Bai and Hui Wang) performed the literature selection independently, and a third author helped resolve any discrepancies if encountered. First, the titles/abstracts of all retrieved articles were reviewed, and studies that did not meet the inclusion criteria were excluded. Then, to determine if the articles meet final eligibility criteria, each author further reviewed the full text of the remaining studies.

\section{Data Extraction}

Two independent authors (Yan Bai and Hui Wang) extracted the relevant information as follows: (1) first authors; (2) publication year; (3) sample size; (4) participant's age; (5) daily step counts; (6) assessment tool. We tried to contact the corresponding author for unclear data to determine whether to include this literature. Any disagreement about data extraction was resolved through consulting a third author.

For quantitative analysis, the mean, standard deviation (or standard error), and sample size were extracted from the included article. When results were presented in figures, data were extracted using the GetData software (getdata-graph-digitizer.com) (9-11). When results were described in terms of median, quartiles (or range) (12-20), data were calculated using the formula proposed by Luo et al. and Wan et al. (https://www.math.hkbu.edu.hk/ tongt/papers/ median2mean.html) $(21,22)$. If data were reported for subgroups (e.g., male vs. female) (23-25), we calculated a pooled mean (standard deviation) by pooling the data of the two different subgroups (26). Furthermore, if a study reported results for different stages of CKD separately, both data were included in the meta-analysis.

\section{Risk of Bias}

The quality of cross-sectional and longitudinal studies was assessed using the JBI Meta-Analysis of Statistics Assessment 
and Review Instrument (JBI-MAStARI) (27). The JBI-MAStARI includes eight questions. Two authors (Yan Bai and Hui Wang) performed the quality assessment procedure independently, with disagreements discussed by both authors or with the help of a third author.

\section{Data Synthesis and Statistical Analysis}

Quantitative data synthesis was used to present the data extracted from each study. Given the potential heterogeneity across the included studies, a random-effects model was used with the DerSimonian-Laird method. Subgroup analysis explored differences in outcomes by stage of CKD.

Heterogeneity between studies was assessed using the $\chi^{2}$ test of Cochrane's Q statistic, and I-square estimates $>75 \%$ were considered highly heterogeneous (28). Contour-enhanced funnel plots, the Egger's test, and the trim-and-fill method were conducted to investigate publication bias across studies.

Univariate and multivariate meta-regression were conducted to examine possible sources of heterogeneity using the following variables: region (Asia, Europe, and America), stage of CKD (predialysis, peritoneal dialysis, hemodialysis, and kidney transplant recipients), samples ( $>100$ and $\leq 100$ ), bias (score $<7$ and $\geq 7$ ), age $(<60$ and $\geq 60$ ), and measurement tools (Accelerometer, Armband, Pedometer, and Fitbit).

$P<0.05$ was considered statistically significant. Stata software (version 12.0) was used to analyze the data.

\section{RESULTS}

\section{Search Results}

The initial search yielded 464 articles and abstracts, and 198 pieces were excluded due to duplication. After screening titles and abstracts, 196 irrelevant articles were excluded. The remaining 36 articles were searched in full text for review based on inclusion and exclusion criteria. Eight articles were excluded, and the reasons for exclusion were listed in Supplementary Material 2. Finally, 28 articles were identified and used for quantitative analysis (9-20, 23-25, 29-41). The flow chart is based on PRISMA 2020 Flow Diagram (8) (Figure 1).

\section{Study Characteristics and Participants}

Hemodialysis patients were the most reported population. Study sample sizes ranged from 20 to 1,163 (Table 1), with a total

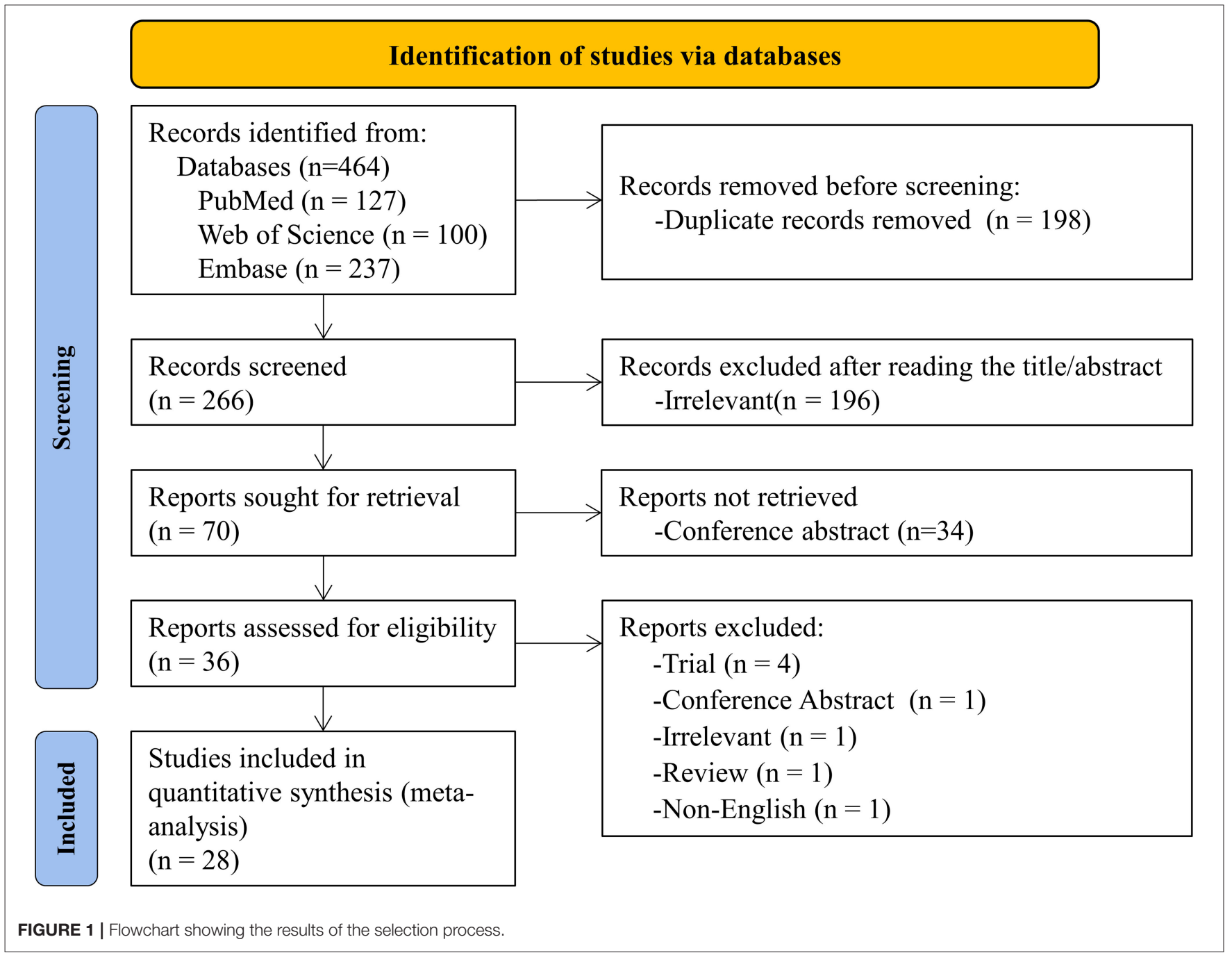


TABLE 1 | Characteristic of observation studies included in the meta-analysis.

\begin{tabular}{|c|c|c|c|c|c|c|}
\hline References & Design & Sample size & Participant's age (years) & Stage of CKD & Measurement & Daily step counts \\
\hline Cupisti et al. (29) & Cross-sectional study & 50 (32 male; 18 female) & $59 \pm 13$ & $H D$ & SenseWear ${ }^{\mathrm{TM}}$ Armband & $\begin{array}{l}\text { HD: } 5,584 \pm 3,734 \\
\text { Normal subjects: } 11,735 \pm 5,130\end{array}$ \\
\hline Matsuzawa et al. (15) & Cross-sectional study & 116 (58 male; 58 female) & $68(62,74)$ & $H D$ & Pedometer & $3,208(1,828,4,481)$ \\
\hline Cobo et al. (30) & Cross-sectional study & $\begin{array}{l}78 \text { HD ( } 51 \text { male; } 27 \text { female) } \\
64 \text { PD ( } 45 \text { male; } 19 \text { female) }\end{array}$ & $\begin{array}{l}\text { HD: } 63 \pm 12 \\
\text { PD: } 62 \pm 14\end{array}$ & $\mathrm{PD}$ and $\mathrm{HD}$ & Pedometer & $\begin{array}{l}\text { HD day: } 2,274 \pm 2,048 \\
\text { Non-HD day: } 3,769 \pm 3,370 \\
\text { PD: } 4,839 \pm 3,313\end{array}$ \\
\hline Brys et al. (18) & Cross-sectional study & 37 & $63 \pm 18$ & $H D$ & SenseWear ${ }^{\mathrm{TM}}$ Armband & 2,424 (892, 4,545) \\
\hline Sheshadri et al. (13) & Cross-sectional study & 48 (39 male; 9 female) & $57(52,65)$ & $\mathrm{HD}$ & Pedometer & $2,631(1,125,5,278)$ \\
\hline Cobo et al. (25) & Cross-sectional study & 57 & $65(49,80)$ & $H D$ & $\begin{array}{l}\text { Geonaute-onstep- } 400^{\circledR} \\
\text { pedometer }\end{array}$ & $\begin{array}{l}\text { Normal testosterone: 4,291 } \pm 3,225 \\
\text { Hypogonadism: 2,753 } \pm 1,784\end{array}$ \\
\hline Yamamoto et al. (31) & Retrospective cohort study & 512 (299 male; 213 female) & $65.4 \pm 11.7$ & $\mathrm{HD}$ & Accelerometer & $\begin{array}{l}\text { Total: } 3,268(1,749-5,195) \\
\text { HD day: } 2,371(1,203-4,240) \\
\text { Non-HD day: } 3,752(1,830-6,271)\end{array}$ \\
\hline Mafra et al. (23) & Cross-sectional study & 24 (18 male; 6 female) & $\begin{array}{l}\mathrm{CRP}<5: 67.0 \pm 14.7 \\
\mathrm{CRP}>5: 69.0 \pm 18.0\end{array}$ & $\mathrm{HD}$ & SenseWear Pro2 Armband & $\begin{array}{l}C R P<5: 6,016 \pm 3,752 \\
C R P>5: 2,801 \pm 2,754 \\
\text { Normal subjects: } 8,107 \pm 5,419 \\
C R P<5: H D \text { day } 4,748 \pm 3,471 \\
C R P<5: \text { Non-HD day } 7,000 \pm 4,670 \\
C R P>5: H D \text { day } 1,810 \pm 1,566 \\
C R P>5: \text { non-HD day } 2,148 \pm 1,780\end{array}$ \\
\hline Dontje et al. (32) & Longitudinal study & 28 (14 male; 14 female) & 54.5 (IQR 15) & KTRs & SenseWear ${ }^{\mathrm{TM}}$ Armband & $6,326 \pm 2,906$ \\
\hline D'Alessandro et al. (24) & Prospective cohort study & 144 (120 male; 24 female) & $\begin{array}{l}\text { Diabetic CKD: } 71.5 \pm 8.2 \\
\text { non-diabetic CKD: } 71.6 \pm 9\end{array}$ & $\begin{array}{l}\text { Stage } 3 b-4 \text { CKD } \\
\text { (eGFR: } 29.8 \pm 9.1 \\
\text { vs. } 31.3 \pm 10.9 \text { ) }\end{array}$ & SenseWear ${ }^{\mathrm{TM}}$ Armband & $\begin{array}{l}\text { Diabetic CKD: } 3,580 \pm 2,471 \\
\text { Non-diabetic CKD: 5,628 } \pm 4,143\end{array}$ \\
\hline Akber et al. (20) & Cross-sectional study & 44 (22 male; 22 female) & $15.1 \pm 3.4$ & $\begin{array}{l}\text { CKD stage 1-4 } \\
\text { [eGFR: } 40.5(27.5, \\
77.0)]\end{array}$ & $\begin{array}{l}\text { Yamax Digi-walker SW-200 } \\
\text { pedometer }\end{array}$ & $6,218(3,637,9,829)$ \\
\hline Panaye et al. (14) & Cross-sectional study & 1163 (762 male; 401 female) & $63(51-75)$ & $\begin{array}{l}\mathrm{HD}(\mathrm{n}=1100) \\
\mathrm{PD}(\mathrm{n}=63)\end{array}$ & Pedometer & $\begin{array}{l}\text { Total: } 3,688(1,866-6,271) \\
\text { HD: } 3,693(1,896-6,307) \\
\text { PD: } 3,320(1,478-5,926) \\
\text { HD day: } 2,912(1,439-5,232) \\
\text { Non-HD day: } 4,054(2,136-7,108)\end{array}$ \\
\hline Avesani et al. (19) & Cross-sectional study & 134 (64 male; 70 female) & $54.9 \pm 15.9$ & $\mathrm{HD}$ & SenseWear Pro2 Armband & $\begin{array}{l}\text { Total: } 5,660(73,16,565) \\
\text { HD day: } 4,620(77,13,957) \\
\text { Non-HD day: } 5,544(72,18,220)\end{array}$ \\
\hline Hamiwka et al. (33) & Cross-sectional study & 20 (8 male; 12 female) & $14.3 \pm 3.2$ & KTRs & $\begin{array}{l}\text { Digi-walker SW200 } \\
\text { pedometer }\end{array}$ & $\begin{array}{l}\text { KTRs: } 9,282 \pm 4,666 \\
\text { Normal subjects: } 11,449 \pm 4,638\end{array}$ \\
\hline Williams et al. (9) & Cross-sectional study & 29 (12 male; 17 female) & $52 \pm 14$ & $\mathrm{HD}$ & $\begin{array}{l}\text { Fitbit }^{\circledR} \text { Flex }^{\mathrm{TM}} \text { tracking } \\
\text { bracelet }\end{array}$ & $\begin{array}{l}\text { HD: } 5,291 \pm 2,338 \\
\text { HD day: } 4351.14 \pm 2266.12 \text { (SE) } \\
\text { Non-HD day: } 6396.91 \pm \\
4909.89 \text { (SE) }\end{array}$ \\
\hline
\end{tabular}


TABLE 1 | Continued

\begin{tabular}{|c|c|c|c|c|c|c|}
\hline References & Design & Sample size & Participant's age (years) & Stage of CKD & Measurement & Daily step counts \\
\hline Lou et al. (34) & Cross-sectional study & 320 (120 male; 200 female) & $58.60 \pm 14.2$ & $H D$ & OMRON pedometer & $3725.92 \pm 2663.47$ \\
\hline Carvalho et al. (35) & Cross-sectional study & $\begin{array}{l}\text { HD: } 20 \text { ( } 11 \text { male; } 9 \text { female) } \\
\text { KTRs: } 23 \text { ( } 11 \text { male; } 12 \\
\text { female) }\end{array}$ & $\begin{array}{l}\text { HD: } 47.3 \pm 12.6 \\
\text { PD: } 48.3 \pm 10.3\end{array}$ & HD KTRs & Triaxial accelerometer & $\begin{array}{l}\text { KTRs: } 9,705 \pm 4,902 \\
\text { HD day: } 6,962 \pm 3,352 \\
\text { Non-HD day: } 4,396 \pm 2,034\end{array}$ \\
\hline Malhotra et al. (11) & Cross-sectional study & 45 (26 male; 19 female) & $61 \pm 15$ & $\mathrm{HD}$ & Fitbit $^{\circledR}$ Charge 2 tracker & $\begin{array}{l}3,688 \pm 2,730 \\
\text { HD day: } 3165.47 \pm 2455.63 \text { (SE) } \\
\text { Non-HD day: } 4124.70 \pm \\
3453.24 \text { (SE) }\end{array}$ \\
\hline Oishi et al. (36) & Cross-sectional study & 38 (22 male; 16 female) & $63.9 \pm 10.8$ & PD & Multi-memory pedometer & $4,367 \pm 2,590$ \\
\hline Han et al. (39) & Cross-sectional study & 29 (16 male; 13 female) & $53 \pm 11$ & $H D$ & Fitbit $^{\circledR}$ Flex & $8,454 \pm 4,087$ \\
\hline Shibata et al. (37) & Cross-sectional study & 24 (13 male; 11 female) & $66.0 \pm 8.2$ & $H D$ & Lifestyle recording device & $\begin{array}{l}\text { HD: } 4,774 \pm 2,845 \\
\text { Normal subjects: } 8,696 \pm 3,047\end{array}$ \\
\hline Katayama et al. (38) & Longitudinal study & 71 (43 male; 28 female) & $70.9 \pm 10.6$ & $H D$ & Tri-accelerometer & $\begin{array}{l}2445.7 \pm 2018.3 \\
\text { HD day: } 2370.6 \pm 2044.6 \\
\text { Non-HD day: } 2502.1 \pm 2236.5\end{array}$ \\
\hline Han et al. (10) & Cross-sectional study & 46 (23 male; 23 female) & $54 \pm 12.9$ & $H D$ & Fitbit $^{\circledR}$ Flex & $\begin{array}{l}\text { Total: } 6,393 \pm 3,550 \\
\text { HD day: } 5642.42 \pm 4369.68 \text { (SE) } \\
\text { Non-HD day: } 6872.73 \pm \\
4581.77 \text { (SE) }\end{array}$ \\
\hline Zhang et al. (12) & Cross-sectional study & 174 (93 male; 81 female) & $63.05 \pm 12.29$ & $H D$ & Digital pocket pedometer & $\begin{array}{l}\text { Low muscle: } 2,803(824,4,154) \\
\text { High muscle: } 5,589(4,659,6,511)\end{array}$ \\
\hline Kittiskulnam et al. (17) & Cross-sectional study & 60 (47 male; 13 female) & $58.0 \pm 12.7$ & $H D$ & Pedometer & $2630.5(1270.7,5137.0)$ \\
\hline Raymond et al. (41) & Cross-sectional study & 32 (14 male; 18 female) & Not reported & KTRs & $\begin{array}{l}\text { Piezo SC-StepMX step } \\
\text { pedometer }\end{array}$ & $9,752 \pm 3,685$ \\
\hline Lunney et al. (16) & Prospective cohort study & 46 (29 male; 17 female) & $64(47,71)$ & $\mathrm{HD}$ & Fitbit tracker & $3,133(1,976,5,097)$ \\
\hline Matsuzawa et al. (40) & Prospective cohort study & 282 (154 male; 128 female) & $64.8 \pm 10.6$ & $\mathrm{HD}$ & Accelerometer & $\begin{array}{l}3,920 \pm 2,797 \\
\text { HD day: } 3,099 \pm 4,337 \\
\text { Non-HD day: } 4,337 \pm 3,160\end{array}$ \\
\hline
\end{tabular}

CKD, Chronic Kidney Disease; PD, Peritoneal Dialysis; HD, Hemodialysis; KTRs, Kidney Transplant Recipients; CRP, C-reaction Protein. 
Study

ID
$\%$

ES $(95 \% \mathrm{Cl})$

Weight pre-dialysis

D'Alessandro C (2019)

Akber A (2012)

Subtotal $(1-$ squared $=77.2 \%, p=0.036)$

PD

Cobo G (2015)

Panaye M (2015)

Oishi D (2012)

Subtotal $(1-$ squared $=57.1 \%, p=0.097)$

HD

Cupisti A (2011)

Matsuzawa R (2013)

Cobo G (2015)

Cobo G (2015)

Yamamoto S (2021)

Brys ADH (2020)

Sheshadri A (2019)

Cobo G (2017)

Mafra D (2011)

Panaye M (2015)

Avesani CM (2012)

Williams S (2017)

Lou X (2019)

Carvalho EV (2014)

Carvalho EV (2014)

Malhotra R (2021)

Han M (2016)

Shibata S (2014)

Katayama A (2016)

Han, M (2018)

Zhang Q (2020)

Kittiskulnam P (2019)

Lunney M (2021)

Matsuzawa R (2018)

Subtotal $(I-$ squared $=92.2 \%, p=0.000)$

KTRs

Dontje ML (2014)

Raymond J (2016)

Hamiwka LA (2009)

Carvalho EV (2014)

Subtotal $(I-$ squared $=85.4 \%, p=0.000)$

Overall (I-squared $=93.5 \%, p=0.000)$

NOTE: Weights are from random effects analysis

1

$-11708$

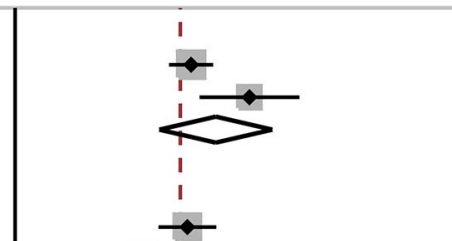

$4945.33(4326.65,5564.01)$

$6583.06(5180.92,7985.20)$

$5638.37(4052.48,7224.25)$

3.35

2.41

5.76

$4839.00(4027.32,5650.68)$

$3589.76(2781.26,4398.26)$

$4367.00(3543.52,5190.48)$

3.14

3.14

3.12

$4264.08(3546.29,4981.87)$

9.40

$5584.00(4549.00,6619.00) \quad 2.86$

$3170.37(2807.95,3532.79) \quad 3.57$

$3769.00(3021.12,4516.88) \quad 3.21$

$2274.00(1819.50,2728.50) \quad 3.50$

$3410.96(3189.05,3632.87) \quad 3.65$

$2632.54(1842.75,3422.33) \quad 3.16$

$3034.99(2137.11,3932.87) \quad 3.03$

$3751.35(3002.80,4499.90) \quad 3.21$

$4810.38(3327.67,6293.09) \quad 2.31$

$3979.09(3785.60,4172.58) \quad 3.66$

$5905.16(5368.25,6442.07) \quad 3.43$

$5291.00(4440.06,6141.94) \quad 3.09$

$3725.92(3434.10,4017.74) \quad 3.62$

$4396.00(3504.57,5287.43) \quad 3.04$

$6962.00(5492.95,8431.05) \quad 2.33$

$3688.00(2890.37,4485.63) \quad 3.15$

$8454.00(6966.50,9941.50) \quad 2.31$

$4774.00(3635.79,5912.21) \quad 2.73$

$2445.70(1976.23,2915.17) \quad 3.49$

$6393.00(5367.12,7418.88) \quad 2.87$

$4083.90(3876.46,4291.34) \quad 3.66$

$3035.57(2292.47,3778.67) \quad 3.22$

$3418.87(2728.69,4109.05) \quad 3.27$

$3920.00(3593.55,4246.45) \quad 3.60$

$4111.82(3760.87,4462.77) \quad 75.98$

$6326.00(5249.63,7402.37) \quad 2.81$

$9752.00(8475.24,11028.76) \quad 2.56$

$9282.00(7237.07,11326.93) \quad 1.73$

$9705.00(7701.64,11708.36) \quad 1.77$

$8689.83(6702.46,10677.20) \quad 8.87$

$4642.47(4274.18,5010.76) \quad 100.00$

FIGURE 2 | Forest plot of the overall step counts.

sample size of 3,769 . The age range across the studies was $15.1-$ 70.9 years. Two studies used a longitudinal design, and 22 were cross-section studies, and four were cohort studies. The details of the quality assessment are shown in Supplementary Material 3.

\section{Overall Daily Step Counts Among Patients With CKD}

Figure 2 shows a forest plot of studies included in the metaanalysis. The results based on the random-effects model showed an overall step count of 4642.47 (95\% CI 4274.18-5010.76) for patients with CKD. The considerable heterogeneity $\left(I^{2}=93.5 \%\right)$ may be a specific result of differences in patient age, stage of disease, and sample size.

Subgroup analysis was performed to determine the overall step counts in different disease stages. Two studies reported step counts for pre-dialysis (5638.37, 95\% CI 4052.48-7224.25) patients. Three and 22 studies reported step counts for peritoneal dialysis (4264.08, 95\% CI 3546.29-4981.87) and hemodialysis 
Study

ID
$\%$

ES $(95 \% \mathrm{Cl})$
Weight

\begin{tabular}{|c|c|c|}
\hline \multirow{2}{*}{$\begin{array}{l}\text { Non-HD day } \\
\text { Panaye M (2015) }\end{array}$} & & \\
\hline & $4451.80(4233.69,4669.90)$ & 8.67 \\
\hline Avesani CM (2012) & $5876.10(5285.29,6466.91)$ & 7.99 \\
\hline Williams S (2017) & $6396.91(-3226.30,16020.12)$ & 0.32 \\
\hline Malhotra R (2021) & $4124.70(-2643.53,10892.93)$ & 0.62 \\
\hline Katayama A (2016) & $2502.10(1981.89,3022.31)$ & 8.15 \\
\hline Han, M (2018) & $6872.73(-2107.37,15852.83)$ & 0.37 \\
\hline Cobo G (2015) & $3769.00(3021.12,4516.88)$ & 7.57 \\
\hline Carvalho EV (2014) & $4396.00(3504.57,5287.43)$ & 7.16 \\
\hline Yamamoto S (2021) & $3961.18(3675.20,4247.16)$ & 8.58 \\
\hline Subtotal $(I-$ squared $=90.2 \%, p=0.000)$ & $4179.70(3490.25,4869.15)$ & 49.44 \\
\hline . & & \\
\hline HD day & & \\
\hline Panaye M (2015) & $3208.60(3042.22,3374.98)$ & 8.71 \\
\hline Avesani CM (2012) & $4841.00(4389.13,5292.87)$ & 8.30 \\
\hline Williams S (2017) & $4351.14(-90.37,8792.65)$ & 1.32 \\
\hline Malhotra R (2021) & $3465.47(-1347.48,8278.42)$ & 1.15 \\
\hline Katayama A (2016) & $2370.60(1895.01,2846.19)$ & 8.25 \\
\hline Han, M (2018) & $5642.42(-2922.00,14206.84)$ & 0.40 \\
\hline Cobo G (2015) & $2274.00(1819.50,2728.50)$ & 8.29 \\
\hline Carvalho EV (2014) & $6962.00(5492.95,8431.05)$ & 5.44 \\
\hline Yamamoto S (2021) & $2616.62(2421.05,2812.19)$ & 8.69 \\
\hline Subtotal $(I-$ squared $=93.8 \%, p=0.000)$ & $3482.40(2819.24,4145.57)$ & 50.56 \\
\hline . & & \\
\hline Overall $(I-$ squared $=95.3 \%, p=0.000)$ & $3862.44(3307.70,4417.19)$ & 100.00 \\
\hline NOTE: Weights are from random effects analysis & & \\
\hline $\begin{array}{c}1 \\
-16020\end{array}$ & & \\
\hline
\end{tabular}

FIGURE 3 | Forest plot of the overall step counts by dialysis and non-dialysis day.

patients (4111.82, 95\% CI 3760.87-4462.77), respectively. Furthermore, four studies reported step counts for kidney transplant recipients (8689.83, 95\% CI 6702.46-10677.20).

\section{Daily Step Counts on Dialysis Days vs.} Non-dialysis Days in Hemodialysis Patients Ten studies reported steps on dialysis days and non-dialysis days in hemodialysis patients (Figure 3). A pooled analysis showed that the overall step counts on a dialysis day was 3413.24 (95\% CI 2825.61-4000.88), with high heterogeneity $\left(I^{2}\right.$ $=93.1 \%)$; while the overall step counts on a non-dialysis day was 4197.83 (95\% CI 3631.92-4763.75), also with high heterogeneity (I-squared $=89.0 \%)$.

\section{Patients With CKD vs. Healthy Controls}

Only four studies (three hemodialysis patients and one kidney transplant recipient) evaluated step counts in patients with CKD compared with healthy controls (Figure 4). A random-effects model showed that step counts were lower in CKD patients than healthy controls (Mean difference: -4034.10 ; 95\% CI -5662.73 to -2405.46 ), and the difference being statistically significant.

\section{Meta-Regression to Explore Heterogeneity for Step Counts in Patients With CKD}

Table 2 shows the results of univariate and multivariate analyses. Univariate analyses showed that the daily step counts were higher across studies with those carried out in the Americas and among the kidney transplant recipients. Patients were aged $<60$ years had significantly higher step counts than patients aged $\geq 60$ years. Therefore, these three variables (region, stage of CKD, and age) were eligible for inclusion in the multivariable regression analysis.

The multivariable model was statistically significant $\left[R^{2}=\right.$ $\left.51.12 \% ; F_{(6,25)} ; P<0.001\right]$ and reduced the $I^{2}$ from 93.5 


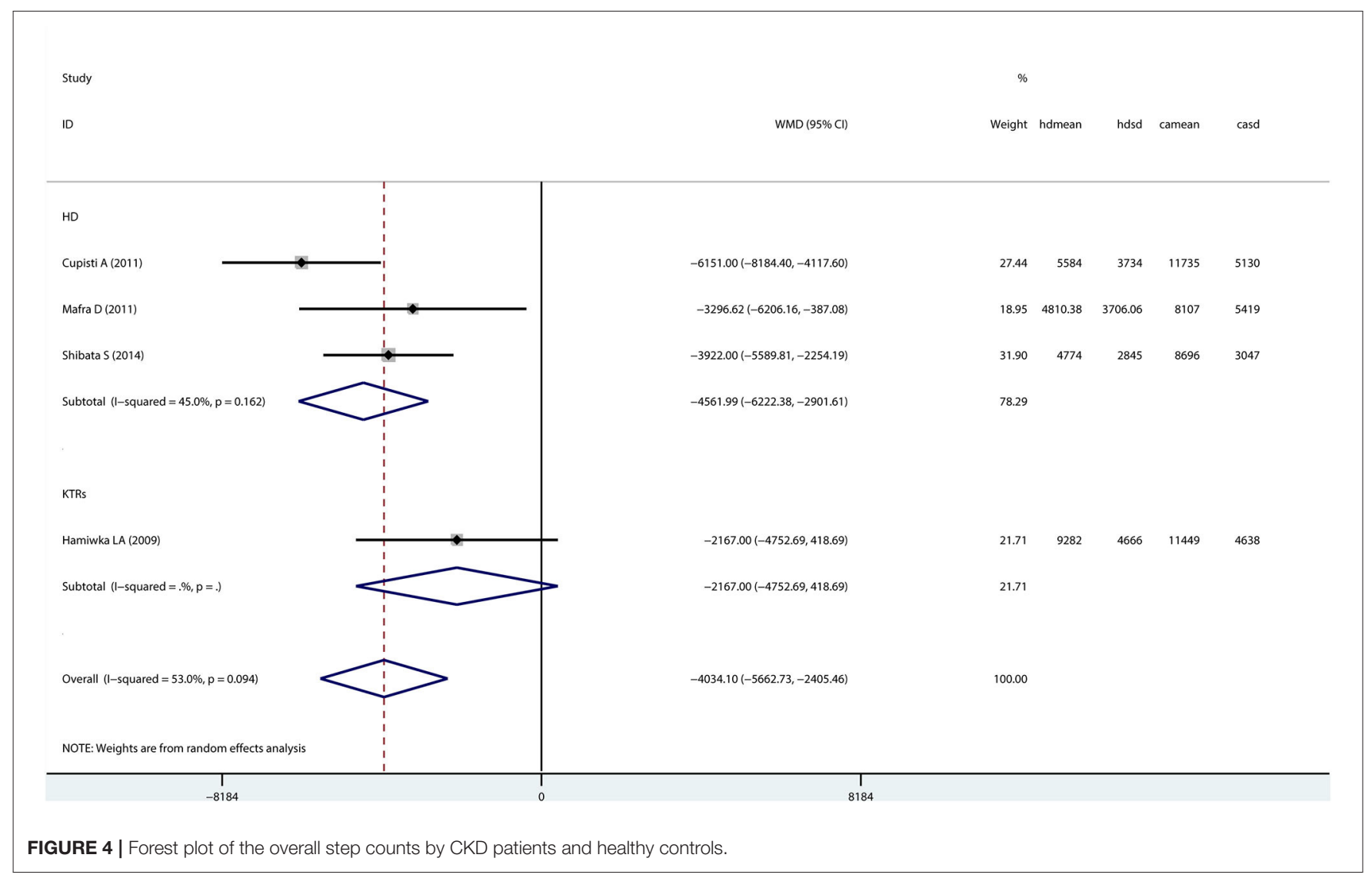

to $89.5 \%$. However, there were no variables that remained statistically significant.

\section{Publication Bias}

As shown in Supplementary Material 4, the contour-enhanced funnel plot was asymmetric. The Egger's test indicates a publication bias $(z=3.67, P=0.004$, Supplementary Material 5 provides the Egger's publication bias plot). Although it is usually impossible to know the precise mechanism for funnel plot asymmetry, publication bias could explain the presence of an asymmetrical funnel plot (26). No significant change in effect size was found when reanalyzing publication bias using the trim-and-fill computation (Supplementary Material 6).

\section{DISCUSSION}

To our knowledge, this is the first systematic review of the literature to examine daily step counts in patients with the full spectrum of CKD. The key findings including (1) daily step counts in patients with CKD was 4642.47 (95\% CI: 4274.185010.76); (2) patients with CKD have significantly lower daily step counts than healthy population, and the step counts began to decrease before dialysis, drops to a freezing point at the hemodialysis phase, and increases after kidney transplantation (Figure 5); (3) relatively high daily step counts in cases in the Americas or younger than 60 or kidney transplant recipients.
Physical activity is a cornerstone of renal rehabilitation in patients with CKD (42). Evidence suggests that regular physical activity is beneficial at all stages of CKD, including improvements in physical fitness, muscle functions, and health-related quality of life (43). More importantly, higher levels of physical activity are associated with a reduced risk of cardiovascular disease, allcause mortality in this population (44). In comparing subjective methods (e.g., diaries, questionnaires) for physical activity assessment, activity monitors are regarded as the gold standard in detecting daily step counts and quantifying the volume of physical activity (45). A survey that included 5,656 participants reported that most CKD patients tend to lead sedentary and inactive lifestyles (46). A significantly higher risk of premature death has been reported in patients with $<4,000$ steps per day compared to hemodialysis patients with more than 4,000 steps per day (HR: 2.37; 95\% CI 1.22-4.60) (40). A recent doseresponse meta-analysis showed a $12 \%$ reduction in the risk of all-cause mortality for every 1,000 increase in step count (HR: 0.88; 95\% CI 0.83-0.93) (47). However, most of the currently published studies were limited to assessing daily step counts taken by patients with CKD at a certain stage, and it would be helpful to pool different studies to understand trends to develop countermeasures to increase their physical activity.

In the current study, the average daily step counts for patients with CKD was 4642.47 (95\% CI 4274.18-5010.76). The global physical activity recommendations issued by the 
TABLE 2 | Univariable and multivariable meta-regression analysis of step count in patients with CKD.

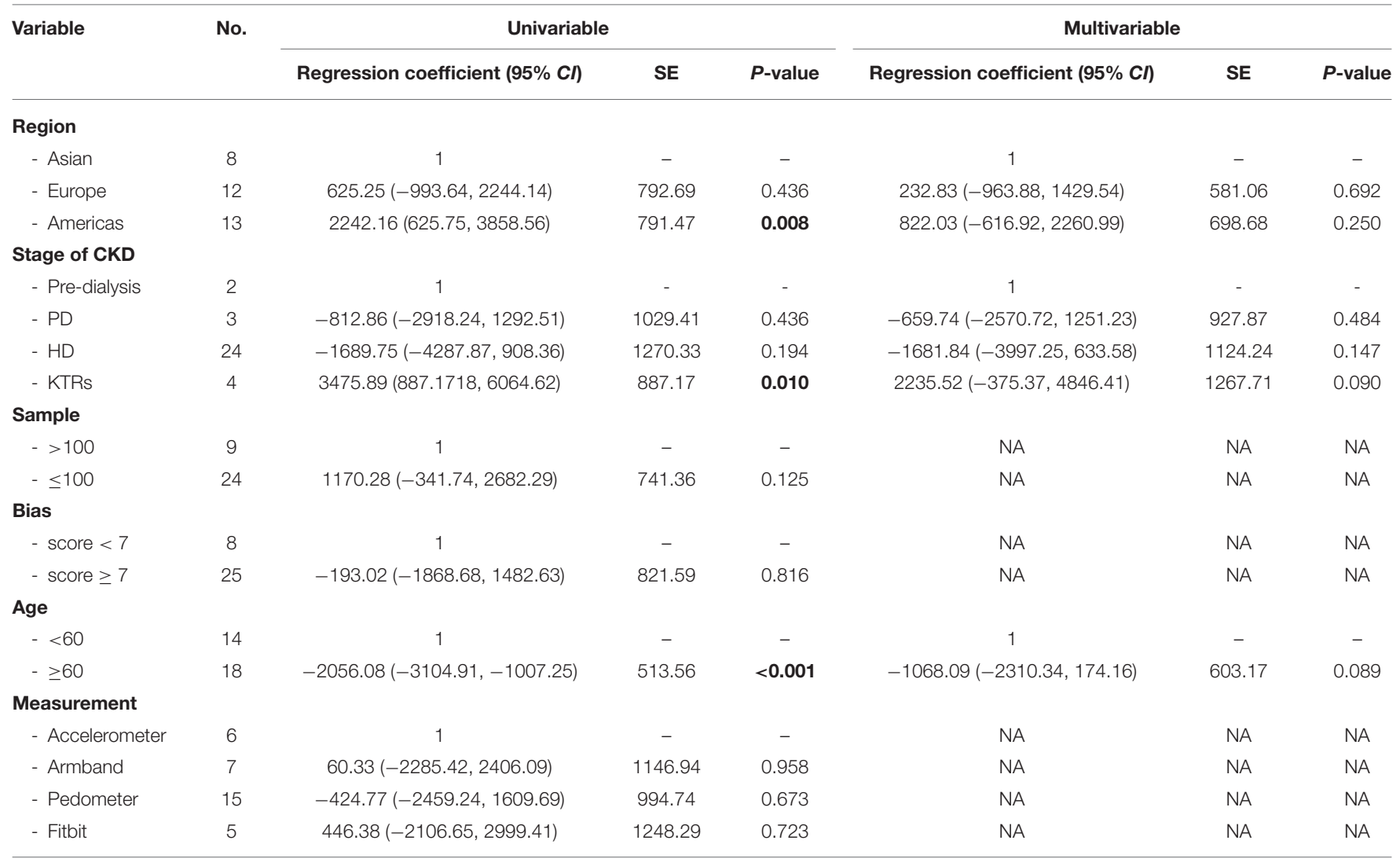

CKD, Chronic Kidney Disease; PD, Peritoneal Dialysis; HD, Hemodialysis; KTRs, Kidney Transplant Recipients; Cl, Confidence Interval; SE, Standard Error; NA, Not Assess. Bold values indicate that the $p$-value $<0.05$.

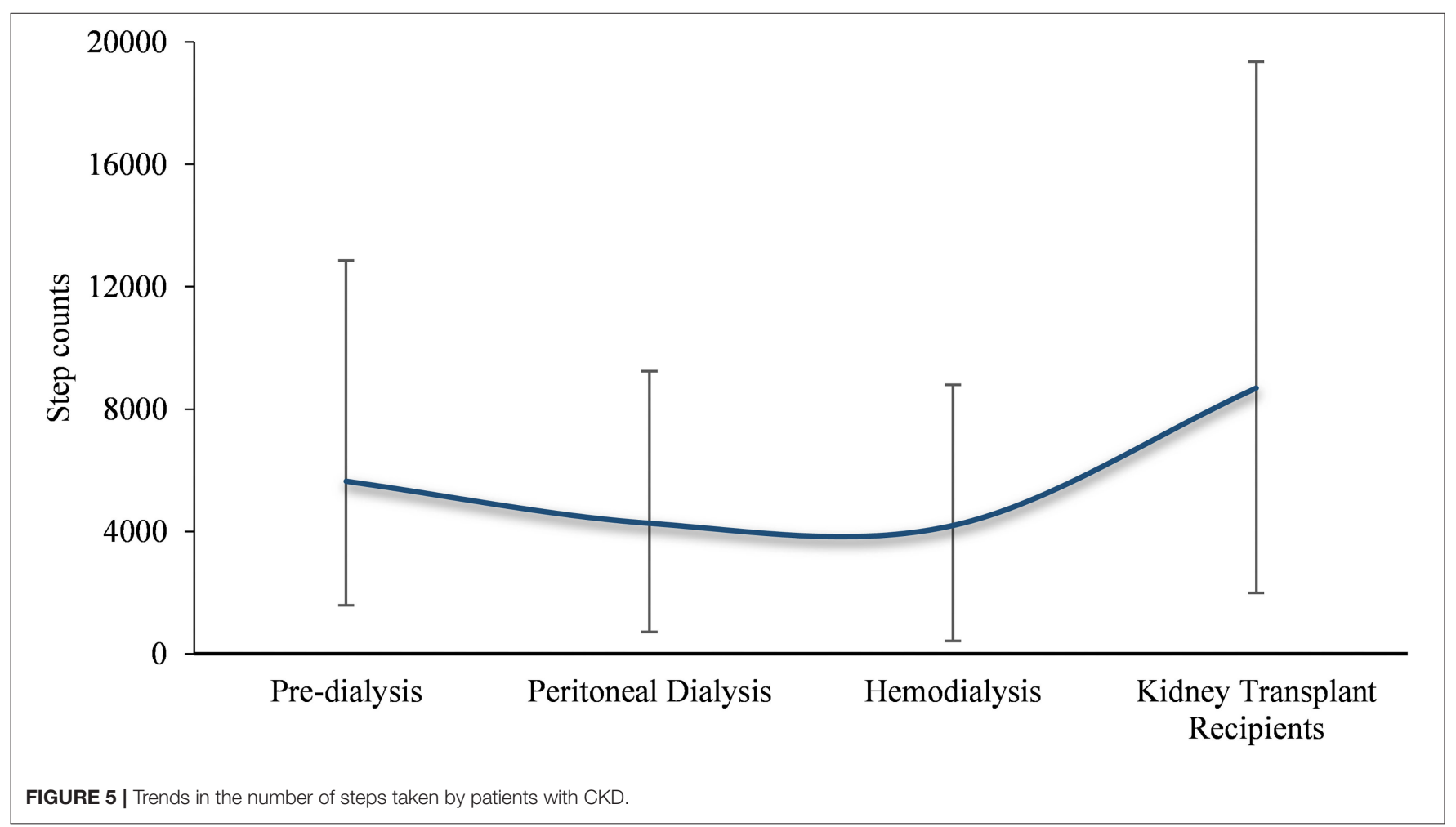


TABLE 3 | A summary of randomized control trials on increasing daily step counts in patients with CKD.

\begin{tabular}{|c|c|c|c|}
\hline References & Stage of CKD & Intervention & Findings \\
\hline Sheshadri et al. (61) & Hemodialysis & $\begin{array}{l}\text { Providing pedometers in conjunction with } \\
\text { weekly semi-scripted counseling sessions, } \\
\text { patients were also set a goal of increasing their } \\
\text { step count by } 10 \% \text { compared to the previous } \\
\text { week. }\end{array}$ & $\begin{array}{l}\text { After } 3 \text { months, patients in the intervention increased their average } \\
\text { daily steps by } 2,256(95 \% \mathrm{Cl}, 978-3,537) \text { more than the controls } \\
(P<0.001) \text {. }\end{array}$ \\
\hline $\begin{array}{l}\text { Assawasaksakul et al. } \\
\text { (62) }\end{array}$ & Hemodialysis & $\begin{array}{l}\text { A } 6 \text {-month intradialytic cycle ergometer for } \\
60 \text { min. }\end{array}$ & $\begin{array}{l}\text { The physical activity in the exercise group was significantly } \\
\text { increased from } 5,613 \text { to } 8,725.1 \text { steps/day in the sixth month }(P= \\
\text { 0.046). }\end{array}$ \\
\hline Martins et al. (63) & Hemodialysis & $\begin{array}{l}\text { A } 12 \text {-weeks moderate-intensity intradialytic } \\
\text { resistance training }\end{array}$ & $\begin{array}{l}\text { After } 12 \text { weeks, patients in the exercise group increased their } \\
\text { average daily steps by } 1,457.8(95 \% \mathrm{Cl},-232.6 \text { to } 3,148.2) \text { than } \\
\text { the controls }(P=0.22) \text {. }\end{array}$ \\
\hline Pecoits et al. (64) & Hemodialysis & $\begin{array}{l}\text { Patients receive high-volume online } \\
\text { hemodiafiltration (HDF) or HD }\end{array}$ & $\begin{array}{l}\text { Patients received HDF was }+538(95 \% \mathrm{Cl},-330 \text { to } 1,407) \\
\text { steps/24 h compared with } \mathrm{HD} \text {, but no statistically significant }(P= \\
\text { 0.262). }\end{array}$ \\
\hline O’Brien et al. (65) & KTRs & $\begin{array}{l}\text { A multicomponent intervention combined } \\
\text { SystemCHANGE }\end{array}$ & $\begin{array}{l}\text { The intervention group increased daily steps at } 3 \text { months (mean } \\
\text { difference, } 608 \text {; standard error }=283, P=0.03 \text { ) compared to the } \\
\text { control group. }\end{array}$ \\
\hline Lyden et al. (66) & Predialysis & $\begin{array}{l}\text { Patients received a Sit Less, Interact, Move } \\
\text { More intervention. }\end{array}$ & $\begin{array}{l}\text { Patients in the intervention group observed a significant increase in } \\
\text { daily steps at week } 20(1265 ; 95 \mathrm{Cl}, 518-2012) \text { but attenuated at } \\
\text { week } 24\end{array}$ \\
\hline
\end{tabular}

American College of Sports Medicine (48) and the World Health Organization (49) recommend $\sim 30$ min of moderate physical activity per day as the minimum physical activity target value for maintaining and promoting health. Walking is recommended as the most basic physical activity for people with all types of chronic diseases. As a recommendation, Tudor-Locke et al. translated the above-recommended values $(30 \mathrm{~min}$ or more of moderate physical activity per day) into a daily step count and recommended that patients with chronic diseases walk at least $7,000-10,000$ steps per day (50). It can be seen that the physical activity level of CKD patients is much lower than recommended. Furthermore, a study by Chen et al. examined the relationship between walking and survival and renal replacement therapy in patients with $\mathrm{CKD}$, reporting a $33 \%$ lower overall risk of death (HR: $0.67 ; 95 \% C I, 0.53-0.84$ ) and a $21 \%$ lower risk of RRT (HR: 0.79; 95\% CI, 0.73-0.85) (5). Additional studies have shown that walking is associated with an improved renal function (OR: 2.23; 95\% CI, 1.91-2.60) in patients with CKD (51) and an inflammatory environment (52).

From the subgroup analysis of this study, the pooled analysis results further clarified the trend in the number of daily steps in patients with $\mathrm{CKD}$ at different stages, i.e., post-transplantation $>$ pre-dialysis $>$ peritoneal dialysis $>$ hemodialysis. This result is similar to the findings of Wilkinson et al. on physical activity in patients with the whole spectrum of CKD (46). Predictably, physical activity levels increase with progressive physical function after kidney transplantation (53); for hemodialysis patients, the combination of dialysis-related fatigue, muscle atrophy, and other comorbidities (e.g., shortness of breath) further decreases physical activity over time in such patients (54). Only renal transplant recipients obtained daily step counts roughly equivalent to the recommended value, indirectly reflecting that most CKD patients do not reach the WHO recommended physical activity level. Therefore, further population-based research is needed to assess the impact of different measures on increasing walking to improve the prognosis of patients with CKD, which should be a priority in renal rehabilitation.

Moreover, elderly patients with CKD have a lower step count and should be prioritized. Frailty is a common comorbidity in CKD patients, and its prevalence increases with age (55). Data support the concept of a spiral of frailty, whereby elder patients with CKD are predisposed to inactivity and frailty (56), and frailty can cause further inactivity and disability (57). However, while disease and aging contribute to frailty, there are also potentially reversible factors, such as inflammation (58). Evidence that increased walking improves indicators of inflammation is wellestablished (59). Walking reflects physical activity in daily life in the elderly population (60); therefore, low step counts in elderly CKD patients suggest the urgency and need for further strengthening of walking management in this population.

We also noted that the assessment of step counts in CKD patients was mostly limited to the dialysis population, largely ignoring the pre-dialysis group, even though there were indications of physical inactivity already in the early stages of $\mathrm{CKD}$. In this regard, of the 28 publications included, only four studies assessed step counts in pre-dialysis patients. Moreover, compared to healthy controls, step counts were at a disadvantage in dialysis patients and kidney transplant recipients. This result is expected, with a reduced step count associated with disease progression.

Based on a search of the literature, we obtained randomized control trials on increasing daily step counts in patients with CKD (Table 3). Overall, feasible strategies included: (1) pedometer-based intervention based on goal-setting theory; (2) physical activity increase based on exercise interventions to improve physical function; (3) changing the dialysis model. 
Different interventions can achieve their effect through different mechanisms of action.

There are some shortcomings of this study that should be mentioned. First, like other meta-analysis $(67,68)$, the statistical heterogeneity of the present meta-analysis was high and remained in the subgroup analysis, which is difficult to avoid in a meta-analysis of observational studies. For this result, we performed a meta-regression, heterogeneity may be associated with region, stage of disease, and age. However, a large portion of the high heterogeneity remains unexplained. Several other factors, such as duration of CKD disease, gender, etc., remain largely unknown and may be responsible for this unexplained heterogeneity. Secondly, although we conducted an exhaustive search of the three primary databases, the lack of non-English publications and unpublished studies may have contributed to the publication bias. We tried to account for this phenomenon by trim-and-fill in our analysis, but it remained unexplained. Thirdly, the current meta-analysis focused on literatures retrieved in standard databases, so gray literature and unpublished articles were not included. Although there may be additional studies of CKD patient step counts in the gray literature that may affect the estimation of pooled effects, we focused on the literature published with peer-reviewed outcomes.

\section{CONCLUSION}

The current systematic review reveals the status of daily step counts in patients with $\mathrm{CKD}$, which decreases with increasing CKD severity and increases after kidney transplantation. Although studies have begun to focus on strategies to improve step counts in patients with CKD, the inclusion of participants

\section{REFERENCES}

1. Coresh J. Update on the Burden of CKD. J Am Soc Nephrol. (2017) 28:10202. doi: 10.1681/ASN.2016121374

2. Saint-Maurice PF, Troiano RP, Bassett DR Jr, Graubard BI, Carlson SA, Shiroma EJ, et al. Association of daily step count and step intensity with mortality among US adults. JAMA. (2020) 323:115160. doi: 10.1001/jama.2020.1382

3. Martins P, Marques EA, Leal DV, Ferreira A, Wilund KR, Viana JL. Association between physical activity and mortality in end-stage kidney disease: a systematic review of observational studies. BMC Nephrol. (2021) 22:227. doi: 10.1186/s12882-021-02407-w

4. Clinical Practice Guideline: Exercise and Lifestyle in Chronic Kidney Disease. Available online at: https://renal.org/sites/renal.org/files/Exercise\%20and \%20Lifestyle\%20in\%20CKD\%20clinical\%20practice\%20guideline33_v4_ FINAL_0.pdf (accessed January 26, 2021).

5. Chen IR, Wang S-M, Liang C-C, Kuo H-L, Chang C-T, Liu J$\mathrm{H}$, et al. Association of walking with survival and RRT among patients with CKD stages 3-5. Clin J Am Soc Nephrol. (2014) 9:1183-9. doi: 10.2215/CJN.09810913

6. Bravata DM, Smith-Spangler C, Sundaram V, Gienger AL, Lin $\mathrm{N}$, Lewis $\mathrm{R}$, et al. Using pedometers to increase physical activity and improve health: a systematic review. JAMA. (2007) 298:2296-304. doi: 10.1001/jama.298.19.2296

7. Bohannon RW. Number of pedometer-assessed steps taken per day by adults: a descriptive meta-analysis. Phys Ther. (2007) 87:1642-50. doi: 10.2522/ptj.20060037 and the short follow-up period require further elucidation of the effects of long-term changes in physical activity in this population. In addition, future studies should focus more on step counts in pre-dialysis patients, and changing their physically inactive lifestyle early in the disease is central to slowing the progression of CKD.

\section{DATA AVAILABILITY STATEMENT}

The original contributions presented in the study are included in the article/Supplementary Material, further inquiries can be directed to the corresponding author.

\section{AUTHOR CONTRIBUTIONS}

FZ and YR: conceptualization and data analysis. YB and HW: retrieved literature and data extraction. FZ and LH: writingoriginal draft. LH: writing-review and editing. All authors have read and approved the final draft.

\section{FUNDING}

This study was supported by the Third Batch of Specialist Nurse Education Program of Longhua Hospital (RC-2021-03-04).

\section{SUPPLEMENTARY MATERIAL}

The Supplementary Material for this article can be found online at: https://www.frontiersin.org/articles/10.3389/fmed. 2022.842423/full\#supplementary-material
8. Page MJ, McKenzie JE, Bossuyt PM, Boutron I, Hoffmann TC, Mulrow CD, et al. The PRISMA 2020 statement: an updated guideline for reporting systematic reviews. BMJ. (2021) 372:n71. doi: 10.1136/bmj.n71

9. Williams S, Han M, Ye X, Zhang H, Meyring-Wösten A, Bonner M, et al. Physical activity and sleep patterns in hemodialysis patients in a suburban environment. Blood Purif. (2017) 43:235-43. doi: 10.1159/000452751

10. Han M, Ye X, Preciado P, Williams S, Campos I, Bonner M, et al. Relationships between neighborhood walkability and objectively measured physical activity levels in hemodialysis patients. Blood Purif. (2018) 45:23644. doi: $10.1159 / 000485161$

11. Malhotra R, Kumar U, Virgen P, Magallon B, Garimella PS, Chopra $\mathrm{T}$, et al. Physical activity in hemodialysis patients on nondialysis and dialysis days: prospective observational study. Hemodial Int. (2021) 25:2408. doi: $10.1111 /$ hdi.12913

12. Zhang Q, Zhang J, Zhang W, Wang M, Huang B, Zhang M, et al. Risk factors for decreased upper-limb muscle strength and its impact on survival in maintenance hemodialysis patients. Int Urol Nephrol. (2020) 52:114353. doi: 10.1007/s11255-020-02468-0

13. Sheshadri A, Kittiskulnam P, Johansen KL. Higher physical activity is associated with less fatigue and insomnia among patients on hemodialysis. Kidney Int Rep. (2019) 4:285-92. doi: 10.1016/j.ekir.2018.10.014

14. Panaye M, Kolko-Labadens A, Lasseur C, Paillasseur JL, Guillodo MP, Levannier M, et al. Phenotypes influencing low physical activity in maintenance dialysis. J Ren Nutr. (2015) 25:31-9. doi: 10.1053/j.jrn.2014.07.010

15. Matsuzawa R, Matsunaga A, Kutsuna T, Ishii A, Abe Y, Yoneki K, et al. Association of habitual physical activity measured by an accelerometer 
with high-density lipoprotein cholesterol levels in maintenance hemodialysis patients. Sci World J. (2013) 2013:780783. doi: 10.1155/2013/780783

16. Lunney M, Wiebe N, Kusi-Appiah E, Tonelli A, Lewis R, Ferber R, et al. Wearable fitness trackers to predict clinical deterioration in maintenance hemodialysis: a prospective cohort feasibility study. Kidney Med. (2021) 3:768-75.e761. doi: 10.1016/j.xkme.2021.04.013

17. Kittiskulnam P, Sheshadri A, Johansen KL. Validation of a new physical activity instrument against pedometers among dialysis patients. J Ren Nutr. (2019) 29:498-503. doi: 10.1053/j.jrn.2019.03.081

18. Brys ADH, Bossola M, Lenaert B, Biamonte F, Gambaro G, Di Stasio E. Daily physical activity in patients on chronic haemodialysis and its relation with fatigue and depressive symptoms. Int Urol Nephrol. (2020) 52:195967. doi: 10.1007/s11255-020-02578-9

19. Avesani CM, Trolonge S, Deléaval P, Baria F, Mafra D, Faxén-Irving G, et al. Physical activity and energy expenditure in haemodialysis patients: an international survey. Nephrol Dial Transplant. (2012) 27:24304. doi: $10.1093 /$ ndt/gfr692

20. Akber A, Portale AA, Johansen KL. Pedometer-assessed physical activity in children and young adults with CKD. Clin J Am Soc Nephrol. (2012) 7:720-6. doi: 10.2215/CJN.06330611

21. Luo D, Wan X, Liu J, Tong T. Optimally estimating the sample mean from the sample size, median, mid-range, and/or mid-quartile range. Stat Methods Med Res. (2018) 27:1785-805. doi: 10.1177/0962280216669183

22. Wan X, Wang W, Liu J, Tong T. Estimating the sample mean and standard deviation from the sample size, median, range and/or interquartile range. BMC Med Res Methodol. (2014) 14:135. doi: 10.1186/1471-2288-14-135

23. Mafra D, Deleaval P, Teta D, Cleaud C, Arkouche W, Jolivot A, et al. Influence of inflammation on total energy expenditure in hemodialysis patients. J Ren Nutr. (2011) 21:387-93. doi: 10.1053/j.jrn.2010.09.006

24. D’Alessandro C, Barsotti M, Cianchi C, Mannucci C, Morganti R, Tassi S, et al, Nutritional aspects in diabetic CKD patients on tertiary care. Medicina. (2019) 55:427. doi: 10.3390/medicina55080427

25. Cobo G, Gallar P, Di Gioia C, García Lacalle C, Camacho R, Rodriguez I, et al. Hypogonadism associated with muscle atrophy, physical inactivity and ESA hyporesponsiveness in men undergoing haemodialysis. Nefrologia. (2017) 37:54-60. doi: 10.1016/j.nefro.2016.04.009

26. Deeks JJ, Higgins JPT, Altman DG. Analysing data and undertaking metaanalyses. In: Higgins JPT, Thomas J, Chandler J, Cumpston M, Li T, Page MJ, Welch VA, editors. Cochrane Handbook for Systematic Reviews of Interventions version 6.2 (updated February 2021). Cochrane (2021). Available online at: www.training.cochrane.org/handbook

27. Ma L-L, Wang Y-Y, Yang Z-H, Huang D, Weng H, Zeng X-T. Methodological quality (risk of bias) assessment tools for primary and secondary medical studies: what are they and which is better? Mil Med Res. (2020) 7:7. doi: 10.1186/s40779-020-00238-8

28. Higgins JPT, Thompson SG. Quantifying heterogeneity in a meta-analysis. Stat Med. (2002) 21:1539-58. doi: 10.1002/sim.1186

29. Cupisti A, Capitanini A, Betti G, D’Alessandro C, Barsotti G. Assessment of habitual physical activity and energy expenditure in dialysis patients and relationships to nutritional parameters. Clin Nephrol. (2011) 75:21825. doi: $10.5414 / \mathrm{CNP} 75218$

30. Cobo G, Gallar P, Gama-Axelsson T, Di Gioia C, Qureshi AR, Camacho $\mathrm{R}$, et al. Clinical determinants of reduced physical activity in hemodialysis and peritoneal dialysis patients. J Nephrol. (2015) 28:503-10. doi: 10.1007/s40620-014-0164-y

31. Yamamoto S, Matsuzawa R, Hoshi K, Harada M, Watanabe T, Suzuki Y, et al. Impact of physical activity on dialysis and nondialysis days and clinical outcomes among patients on hemodialysis. J Ren Nutr. (2021) 31:3808. doi: 10.1053/j.jrn.2020.07.007

32. Dontje ML, de Greef MH, Krijnen WP, Corpeleijn E, Kok T, Bakker SJ, et al. Longitudinal measurement of physical activity following kidney transplantation. Clin Transplant. (2014) 28:394-402. doi: 10.1111/ctr.12325

33. Hamiwka LA, Cantell M, Crawford S, Clark CG. Physical activity and health related quality of life in children following kidney transplantation. Pediatr Transplant. (2009) 13:861-7. doi: 10.1111/j.1399-3046.2009.01195.x

34. Lou X, Li Y, Shen H, Juan J, He Q. Physical activity and somatic symptoms among hemodialysis patients: a multi-center study in Zhejiang, China. BMC Nephrol. (2019) 20:477. doi: 10.1186/s12882-019-1652-z
35. Carvalho EV, Reboredo MM, Gomes EP, Teixeira DR, Roberti NC, Mendes JO, et al. Physical activity in daily life assessed by an accelerometer in kidney transplant recipients and hemodialysis patients. Transplant Proc. (2014) 46:1713-7. doi: 10.1016/j.transproceed.2014.05.019

36. Oishi D, Koitabashi K, Hiraki K, Imai N, Sakurada T, Konno Y, et al. Physical activity is associated with serum albumin in peritoneal dialysis patients. $A d v$ Perit Dial. (2012) 28:148-52.

37. Shibata S, Tsutou A, Shiotani H. Relation between sleep quality and daily physical activity in hemodialysis outpatients. Kobe J Med Sci. (2014) 59:E161166.

38. Katayama A, Miyatake N, Nishi H, Ujike K, Hashimoto H, Kurato R, et al. Relationship between changes in physical activity and changes in healthrelated quality of life in patients on chronic hemodialysis with 1-year followup. Acta Med Okayama. (2016) 70:353-61. doi: 10.18926/AMO/54593

39. Han M, Williams S, Mendoza M, Ye X, Zhang H, Calice-Silva V, et al. Quantifying physical activity levels and sleep in hemodialysis patients using a commercially available activity tracker. Blood Purif. (2016) 41:194204. doi: $10.1159 / 000441314$

40. Matsuzawa R, Roshanravan B, Shimoda T, Mamorita N, Yoneki K, Harada $\mathrm{M}$, et al. Physical activity dose for hemodialysis patients: where to begin? Results from a Prospective Cohort Study. J Ren Nutr. (2018) 28:4553. doi: 10.1053/j.jrn.2017.07.004

41. Raymond J, Johnson ST, Diehl-Jones W, Vallance JK. Walking, Sedentary Time and health-related quality life among kidney transplant recipients: an exploratory study. Transplant Proc. (2016) 48:59-64. doi: 10.1016/j.transproceed.2015.12.022

42. Hoshino J. Renal rehabilitation: exercise intervention and nutritional support in dialysis patients. Nutrients. (2021) 13:14444. doi: 10.3390/nu13051444

43. Heiwe S, Jacobson SH. Exercise training in adults with CKD: a systematic review and meta-analysis. Am J Kidney Dis. (2014) 64:383-93. doi: 10.1053/j.ajkd.2014.03.020

44. MacKinnon HJ, Wilkinson TJ, Clarke AL, Gould DW, O'Sullivan TF, Xenophontos S, et al. The association of physical function and physical activity with all-cause mortality and adverse clinical outcomes in nondialysis chronic kidney disease: a systematic review. Ther Adv Chronic Dis. (2018) 9:209-26. doi: 10.1177/2040622318785575

45. Yang C-C, Hsu Y-L. A review of accelerometry-based wearable motion detectors for physical activity monitoring. Sensors. (2010) 10:777288. doi: $10.3390 /$ s100807772

46. Wilkinson TJ, Clarke AL, Nixon DGD, Hull KL, Song Y, Burton JO, et al. Prevalence and correlates of physical activity across kidney disease stages: an observational multicentre study. Nephrol Dial Transplant. (2021) 36:6419. doi: $10.1093 / \mathrm{ndt} / \mathrm{gfz} 235$

47. Jayedi A, Gohari A, Shab-Bidar S. Daily step count and all-cause mortality: a dose-response meta-analysis of prospective cohort studies. Sports Med. (2021) 52:89-99. doi: 10.1007/s40279-021-01536-4

48. Haskell WL, Lee IM, Pate RR, Powell KE, Blair SN, Franklin BA, et al. Physical activity and public health: updated recommendation for adults from the American College of Sports Medicine and the American Heart Association. Circulation. (2007) 116:108193. doi: 10.1161/CIRCULATIONAHA.107.185649

49. Global Recommendations on Physical Activity for Health. (2010). Available online at: https://www.who.int/publications/i/item/9789241599979 (accessed January 26, 2021).

50. Tudor-Locke C, Craig CL, Aoyagi Y, Bell RC, Croteau KA, De Bourdeaudhuij I, et al. How many steps/day are enough? For older adults and special populations. Int J Behav Nutr Phys Act. (2011) 8:80. doi: 10.1186/1479-5868-8-80

51. Yu D, Chen Y, Chen T, Cai Y, Qin R, Jiang Z, et al. Walking, but not other physical activity at a higher intensity, is associated with improved kidney function: a cross-sectional health survey of general adult population. J Phys Act Health. (2018) 15:600-4. doi: 10.1123/jpah.2017-0412

52. Viana JL, Kosmadakis GC, Watson EL, Bevington A, Feehally J, Bishop NC, et al. Evidence for anti-inflammatory effects of exercise in CKD. J Am Soc Nephrol. (2014) 25:2121-30. doi: 10.1681/ASN.2013070702

53. Takahashi A, Hu SL, Bostom A. Physical activity in kidney transplant recipients: a review. Am J Kidney Dis. (2018) 72:433-43. doi: 10.1053/j.ajkd.2017.12.005 
54. Delgado C, Johansen KL. Barriers to exercise participation among dialysis patients. Nephrol Dial Transplant. (2012) 27:1152-7. doi: 10.1093/ndt/gfr404

55. McAdams-DeMarco MA, Law A, Salter ML, Boyarsky B, Gimenez L, Jaar BG, et al. Frailty as a novel predictor of mortality and hospitalization in individuals of all ages undergoing hemodialysis. J Am Geriatr Soc. (2013) 61:896-901. doi: 10.1111/jgs.12266

56. Zelle DM, Klaassen G, van Adrichem E, Bakker SJL, Corpeleijn E, Navis G. Physical inactivity: a risk factor and target for intervention in renal care. Nat Rev Nephrol. (2017) 13:152-68. doi: 10.1038/nrneph.2016.187

57. Portilla Franco ME, Tornero Molina F, Gil Gregorio P. Frailty in elderly people with chronic kidney disease. Nefrologia. (2016) 36:60915. doi: 10.1016/j.nefroe.2016.12.005

58. Lorenz EC, Kennedy CC, Rule AD, LeBrasseur NK, Kirkland JL, Hickson LJ. Frailty in CKD and transplantation. Kidney Int Rep. (2021) 6:227080. doi: 10.1016/j.ekir.2021.05.025

59. Carney EF. Chronic kidney disease. Walking reduces inflammation in predialysis CKD. Nat Rev Nephrol. (2014) 10:300. doi: 10.1038/nrneph.2014.75

60. Aggio D, Papachristou E, Papacosta O, et al. Twenty-year trajectories of physical activity types from midlife to old age. Med Sci Sports Exerc. (2019) 51:481-9. doi: 10.1249/MSS.0000000000001802

61. Sheshadri A, Kittiskulnam P, Lazar AA, Johansen KL. A walking intervention to increase weekly steps in dialysis patients: a pilot randomized controlled trial. Am J Kidney Dis. (2020) 75:488-96. doi: 10.1053/ j.ajkd.2019.07.026

62. Assawasaksakul N, Sirichana W, Joosri W, Kulaputana O, Eksakulkla S, Ketanun C, et al. Effects of intradialytic cycling exercise on daily physical activity, physical fitness, body composition, and clinical parameters in high-volume online hemodiafiltration patients: a pilot randomizedcontrolled trial. Int Urol Nephrol. (2021) 53:359-71. doi: 10.1007/ s11255-020-02677-7

63. Martins do Valle F, Valle Pinheiro B, Almeida Barros AA, Ferreira Mendonça W, de Oliveira AC, de Oliveira Werneck G, et al. Effects of intradialytic resistance training on physical activity in daily life, muscle strength, physical capacity and quality of life in hemodialysis patients: a randomized clinical trial. Disabil Rehabil. (2020) 42:3638-44. doi: 10.1080/09638288.2019.1606857

64. Pecoits-Filho R, Larkin J, Poli-de-Figueiredo CE, Cuvello-Neto AL, Barra $\mathrm{ABL}$, Gonçalves PB, et al. Effect of hemodiafiltration on measured physical activity: primary results of the HDFIT randomized controlled trial. Nephrol Dial Transplant. (2021) 36:1057-70. doi: 10.1093/ndt/gfaa173

65. O’Brien T, Russell CL, Tan A, Mion L, Rose K, Focht B, et al. A pilot randomized controlled trial using system $\mathrm{CHANGE}^{\mathrm{TM}}$ approach to increase physical activity in older kidney transplant recipients. Prog Transplant. (2020) 30:306-14. doi: 10.1177/1526924820958 148

66. Lyden K, Boucher R, Wei G, Zhou N, Christensen J, Chertow GM, et al. Targeting sedentary behavior in CKD: a pilot and feasibility randomized controlled trial. Clin J Am Soc Nephrol. (2021) 16:71726. doi: 10.2215/CJN.12300720

67. Fellmeth G, Rose-Clarke K, Zhao C, Busert LK, Zheng Y, Massazza A, et al. Health impacts of parental migration on left-behind children and adolescents: a systematic review and meta-analysis. Lancet. (2018) 392:256782. doi: 10.1016/S0140-6736(18)32558-3

68. Mata DA, Ramos MA, Bansal N, Khan R, Guille C, Di Angelantonio E, et al. Prevalence of depression and depressive symptoms among resident physicians: a systematic review and meta-analysis. JAMA. (2015) 314:237383. doi: 10.1001/jama.2015.15845

Conflict of Interest: The authors declare that the research was conducted in the absence of any commercial or financial relationships that could be construed as a potential conflict of interest.

Publisher's Note: All claims expressed in this article are solely those of the authors and do not necessarily represent those of their affiliated organizations, or those of the publisher, the editors and the reviewers. Any product that may be evaluated in this article, or claim that may be made by its manufacturer, is not guaranteed or endorsed by the publisher.

Copyright (c) 2022 Zhang, Ren, Wang, Bai and Huang. This is an open-access article distributed under the terms of the Creative Commons Attribution License (CC BY). The use, distribution or reproduction in other forums is permitted, provided the original author(s) and the copyright owner(s) are credited and that the original publication in this journal is cited, in accordance with accepted academic practice. No use, distribution or reproduction is permitted which does not comply with these terms. 